\title{
Characterization and Optimization of Mechanical Properties of ABS Parts Manufactured by the Fused Deposition Modelling Process
}

\author{
Godfrey C. Onwubolu and Farzad Rayegani \\ School of Mechanical and Electrical Engineering \& Technology, Faculty of Applied Science and Technology, \\ Sheridan Institute of Technology and Advanced Learning, Brampton, ON, Canada L6Y 5 H9
}

Correspondence should be addressed to Godfrey C. Onwubolu; godfrey.onwubolu@sheridancollege.ca

Received 7 July 2014; Accepted 10 October 2014; Published 10 November 2014

Academic Editor: Luigino Filice

Copyright (C) 2014 G. C. Onwubolu and F. Rayegani. This is an open access article distributed under the Creative Commons Attribution License, which permits unrestricted use, distribution, and reproduction in any medium, provided the original work is properly cited.

\begin{abstract}
While fused deposition modelling (FDM) is one of the most used additive manufacturing (AM) techniques today due to its ability to manufacture very complex geometries, the major research issues have been to balance ability to produce aesthetically appealing looking products with functionality. In this study, five important process parameters such as layer thickness, part orientation, raster angle, raster width, and air gap have been considered to study their effects on tensile strength of test specimen, using design of experiment (DOE). Using group method of data handling (GMDH), mathematical models relating the response with the process parameters have been developed. Using differential evolution (DE), optimal process parameters have been found to achieve good strength simultaneously for the response. The optimization of the mathematical model realized results in maximized tensile strength. Consequently, the additive manufacturing part produced is improved by optimizing the process parameters. The predicted models obtained show good correlation with the measured values and can be used to generalize prediction for process conditions outside the current study. Results obtained are very promising and hence the approach presented in this paper has practical applications for design and manufacture of parts using additive manufacturing technologies.
\end{abstract}

\section{Introduction}

Stratasys Inc. developed the fused deposition modeling (FDM) system which is one such layered manufacturing technology that produces parts with complex geometries by the layering of extruded materials, such as acrylonitrile butadiene styrene (ABS) thermoplastic $[1,2]$ as shown in Figure 1. In the FDM process, the build material is initially in the raw form of a flexible filament. The feedstock filament is then partially melted and extruded though a heated nozzle within a temperature controlled environment for building of the part. The material is extruded in a thin layer onto the previously built model layer on the build platform in the form of a prescribed two-dimensional $(x-y)$ layer pattern. The deposited material cools, solidifies, and bonds with adjoining material. After an entire layer is deposited, the build platform moves downward along the $z$-axis by an increment equal to the filament height (layer thickness) and the next layer is deposited on top of it. The platen or table on which the build sheet is placed lies on the $x-y$ plane.

The properties of built parts depend on settings of various process parameters fixed at the time of fabrication. Additive manufacturing (AM) is a new manufacturing technology, driven by computer-aided design (CAD) that makes it possible for companies to significantly cut design and manufacturing cycle times [1]. This new manufacturing paradigm is seamless because it has the capability of producing exactly any simple or complex part represented by a CAD system which is submitted to it. This paper first proposes a framework for additive manufacturing. In this framework, there are three main domains that drive AM: CAD-based, AM process planning and technologies, and materials/testing/inductive modeling. The designer chooses a CAD system for modeling from a suite of CATIA, SolidWorks, Inventor, and AutoCAD 3D. Then the design engineer utilizes design of experiment (DOE) for optimized process plan for different options of 


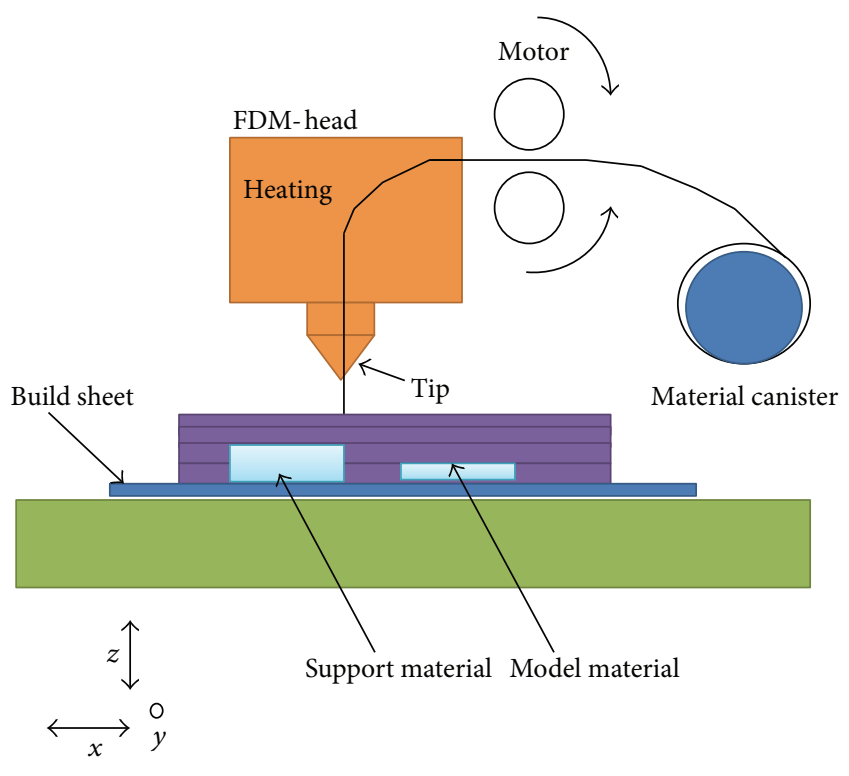

FIGURE 1: FDM process.

a part; intermediate software such as Insight is used for tool paths optimization. A decision has to be made regarding which type of machine to use depending on the material used for producing the part(s). For example, Fortus $900 \mathrm{mc}$ and $400 \mathrm{mc}$ are useful for producing parts made from thermoplastics. Spectrum Z510 are suited for powder materials, while Solidscape suits wax material. These are some of the decisions and choices to be made. The presentation of this paper follows this framework.

Fused deposition modelling (FDM) is a fast growing additive manufacturing (AM) technology due to its ability to build functional parts that have complex geometrical shapes in reasonable build time $[1,2]$. Reduction of product development cycle time is a major concern in industries to remain competitive in the marketplace. Several sources have highlighted the advantages of additive manufacturing techniques such as additive manufacturing (AM) over traditional product development methodology [3-5]. Using AM speeds up the whole product development process especially when producing very complicated parts which may be challenging using traditional manufacturing methods. It is argued that since three-dimensional CAD is being used as the starting point and the transfer to AM is relatively seamless, there is less concern over interpretation of the design intent [1]. The seamlessness can also be seen in terms of reduction in process steps because, regardless of the complexity of parts to be built, building with an AM machine is generally performed in a single step unlike in most other manufacturing processes which normally require multiple and iterative stages to be carried out. In addition to these advantages, other advantages of using AM are significant reduction in the number of processes and resources required. Consequently, focus is fast shifting from traditional product development methodology to additive manufacturing technology [1]. References $[1,2]$ are excellent resources for the processes involved in and advantages of additive manufacturing.
Producing aesthetically appealing AM products that have complex shapes is not difficult, given available AM technologies. The challenge is to produce manufactured AM parts that are functionally reliable. Therefore, this paper reports the work that has been done to investigate the functionality of manufactured AM parts. The motivation for this emphasis is that no company will want to make commitment to a technology that may produce aesthetically appealing products that are not functionally reliable. A critical review of literature suggests that properties of AM parts are a function of various process related parameters and can be significantly improved with proper adjustment. Since mechanical properties are important for functional parts, it is absolutely essential to study the influence of various process parameters on mechanical properties so that improvement can be made through selection of best settings. The present study focuses on assessment of one of the mechanical properties, namely, tensile strength of fused deposition modelling- (FDM-) based fabricated parts, by first developing a model prediction and then optimizing the process parameter settings and responses.

\section{Literature Review}

Although AM is an efficient technology, full scale application has not gained much attention because of compatibility of presently available materials with AM technologies [6]. Despite several design and environmental advantages of AM technologies over other manufacturing processes, the adoption of AM as a means for fabricating end-use components has historically been setback by the technologies' narrow selection of available materials. The majority of materials used presently by modern AM techniques are proprietary polymers. There are also possibilities to use some metals (such as steel alloys and titanium) and ceramics. While there are different types of materials that can be used in $\mathrm{AM}$, the material properties are typically not as strong as their conventionally manufactured counterparts due to the anisotropy caused by the layer-by-layer [7]. To overcome this limitation, one approach may be development of new materials having superior characteristics than conventional materials and its compatibility with technology. Another convenient approach may be suitably adjusting the process parameters during fabrication stage so that properties may improve $[8,9]$. Several researchers have specifically considered the anisotropic characteristics of FDM parts in recent years. Rodríguez et al. [10] investigated the tensile strength and elastic modulus of FDM specimens with varying mesostructures in comparison with the properties of the ABS monofilament feedstock. The outcome of their investigation is that the tensile strength was the greatest for parts with fibers aligned with the axis of the tension force. The mechanical properties of FDM parts are not solely controlled by the build material of the original filament but are also significantly influenced by a directionally dependent production process that fabricates components with anisotropic characteristics associated with the inherent layering [11]. A material in which the elastic properties depend on the orientation of the sample is said to be anisotropic. Ahn et al. [11] designed 
a factorial experiment to quantify the effects of model temperature, bead width, raster orientation, air gap, and acrylonitrile butadiene styrene (ABS) color on both tensile and compressive strengths of FDM parts. They determined that both air gap and raster orientation had significant effects on the resulting tensile strength, while compressive strength was not affected by these factors. Their results include a set of recommended build rules for designing FDM parts. Sood et al. [12] carried out similar study, with varying factors of layer thickness, build orientation, raster angle, raster width, and air gap. They used response surface methodology (RSM) to analyze the functional relationship between process parameters and specimen strength and found that the tested factors influence the mesostructural configuration of the built part as well as the bonding and distortion within the part. Sood et al. [13] further examined the effect of the same five process parameters on the subsequent compressive strength of test specimens. Their results show the importance of fiber-to-fiber bond strength and the control of distortion during the build process. Lee et al. [14] concluded that layer thickness, raster angle, and air gap influence the elastic performance of compliant ABS prototypes manufactured by fused deposition. Lee et al. [15] focused on the compressive strength of layered parts as a function of build direction and determined that the compressive strength is greater for the axial FDM specimens than for the transverse. Panda et al. [16] performed experiments on the impact of process parameters on dimensional accuracy, surface roughness, and mechanical strengths, using response surface methodology (RSM) [17] for modelling and bacterial foraging for finding optimal process parameter settings and responses.

From the literature, it is found that good amount of work has been done in FDM strength modelling; however, little amount of work has been done to develop the strength model in terms of FDM process parameters for prediction purpose. Most published work on predictive model of FDM parts are based on response surface methodology (RSM) which is restrictive in modelling because it is not "datadriven"; that is, it is not inductive. Since the relation between a particular mechanical property and process parameters related to it is difficult to establish, attempt has been made in this paper to derive the empirical model between the processing parameters and mechanical properties using group method of data handling (GMDH) [18] which is one of the most robust existing inductive modelling. The present study reported in this paper uses the group method of data handling $(\mathrm{GMDH})$ modelling approach to derive the required relationship among respective process parameters and tensile strength. The models derived serve as predictive models which can be used to anticipate the theoretical best parameter settings that would result in optimal response characteristic. The predictive models are therefore the objective functions, while the lower and upper bounds used from the design of experiment (DOE) [19] are the constraints, so that the problem becomes constrained optimization which can be solved using any of the existing optimization techniques. In our case, we used differential evolution (DE) [20] to solve the optimization problem. The solutions give the optimal tensile response and optimal process parameter settings.

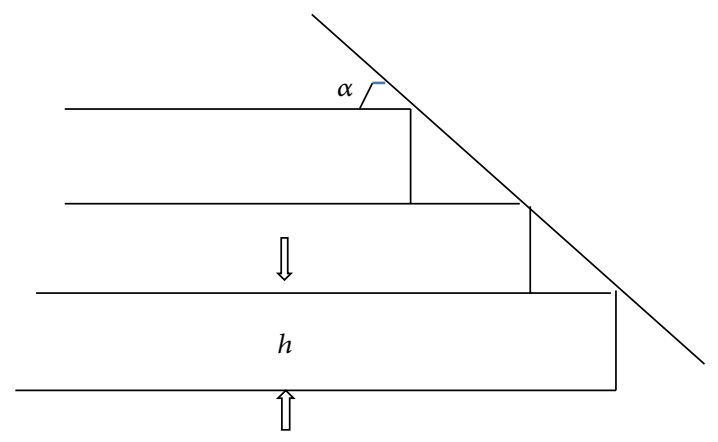

FIgURE 2: Height of slices or layout of layer thickness. Source: [26].

\section{Design of Experiment (DOE) and Experimentation Setup}

In this study, five important process parameters such as layer thickness $(A)$, part orientation $(B)$, raster angle $(C)$, raster width $(D)$, and air gap $(E)$ have been considered to study their effects on tensile strength (TS). ABS material is used; that is, the material is constant. The temperature is also considered constant.

The definitions of FDM variable parameters in this study are as follows.

(A) The layer thickness which is recognized as the height of deposited slice from the FDM nozzle is shown in Figure 2. The layer thickness parameter is used to examine the influence of building thicker or thinner layers on the outcome quality.

(B) The orientation of part is defined as how the part should be positioned when produced as shown in Figure 3 (vertical position (a); inclined position (b); horizontal position (c)).

(C) Raster angle or orientation which is measured from the $x$-axis on the bottom part layer as shown in Figure 4. It also refers to the direction of the beads of material (roads) relative to the loading of the part. The deposited roads can be built at different angles to fill the interior part.

(D) The raster width or road width which refers to the width of the deposition path related to tip size. It also refers to the tool path width of the raster pattern used to fill interior regions of the part curves as shown in Figure 5. Narrow and wide filling pattern (roads) were considered to be examined.

(E) The air gap parameter which is defined as the space between the beads of deposited FDM material is shown in Figure 6. Hence, the influence of applying positive and negative gap between the deposited beads was investigated.

3.1. Experimental Procedure. The 3D models of specimen were modelled in SolidWorks and exported as STL file. The STL file is imported to FDM Insight software (Figure 7). 


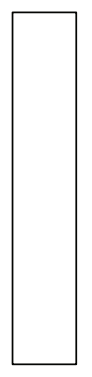

(a)

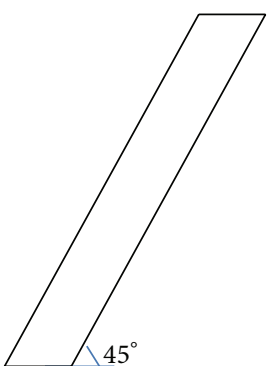

(b)

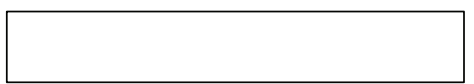

(c)

FIGURE 3: Orientation of part. Source: [11].

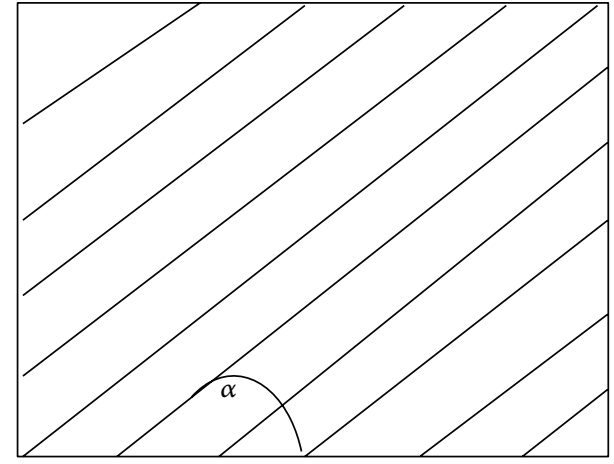

FIgURE 4: Raster angle parameter. Source: [11].

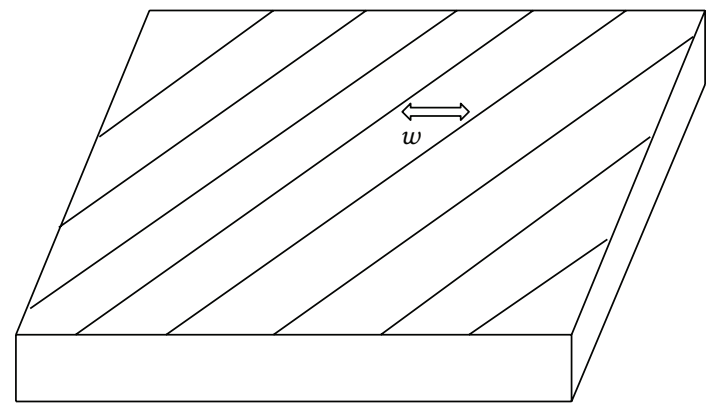

FIGURE 5: Raster width parameter. Source: [11].

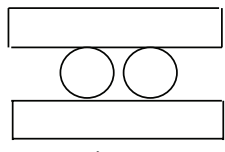

+ air gap

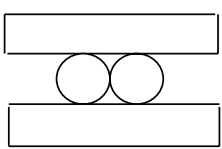

0 air gap

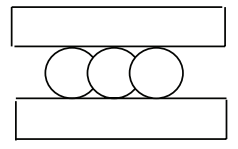

- air gap
FIGURE 6: Air gap application. Source: [11].

Figure 8 reveals the internal structure of the model showing the raster width, raster angle, and air gap. This internal structure was obtained by slicing the model midway along its length for raster angle of $45^{\circ}$, raster width of $0.016^{\prime \prime}$, and air gap of $0.02^{\prime \prime}$ in order to clarify the concepts covered in the earlier part of this section. Understanding of these concepts is important because the design of experiments described in the later part of this section is based on the different settings

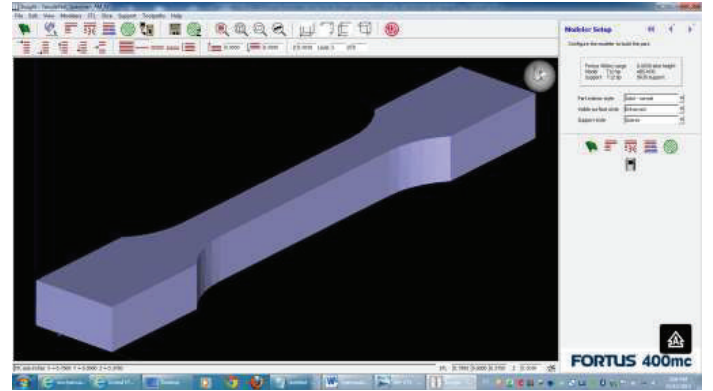

FIGURE 7: STL file of 3D model exported to FDM Insight software.

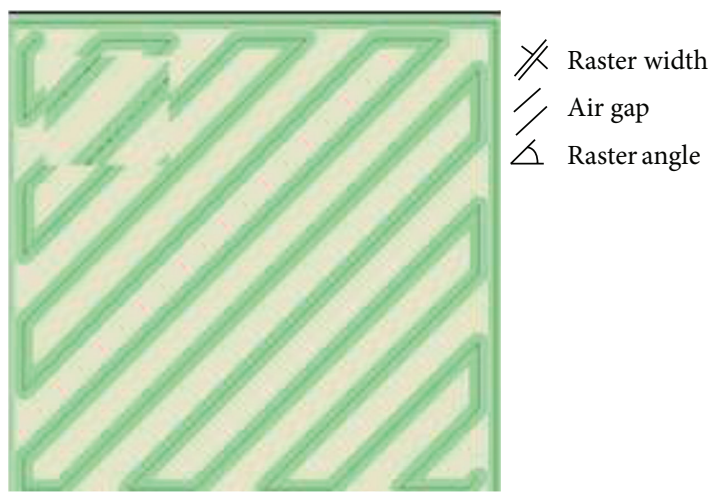

FIGURE 8: Internal structure of the model showing the raster width, raster angle, and air gap.

of the FDM parameters used for our experimentation, which are essentially raster angle, raster width, and air gap together with layer thickness and part orientation.

All testing specimens were constructed in a Stratasys FDM Fortus 400mc System (Figure 9) in the Advanced Manufacturing and Robotics laboratory at Sheridan Institute of Technology. The laboratory is equipped with both Fortus $400 \mathrm{mc}$ and $900 \mathrm{mc}$ Systems. The tensile test was performed using United Testing System (UTS), Model SSTM, Serial 1210555 with capacity of $20 \mathrm{kN}$ (Figure 10) in accordance with ISO R527:1966 and ISO R178:1975, respectively.

3.2. Design of Experiment (DOE). In this work, factors as shown Table 1 are set as per experiment plan (Table 2) 
TABLE 1: Variable process parameters and their selected low and high levels.

\begin{tabular}{lccc}
\hline Variable parameter & Unit & Low level $(-1)$ & High level $(+)$ \\
\hline Layer thickness $(A)$ & in./mm & $0.005^{\prime \prime} /(0.127)$ & $0.013^{\prime \prime} /(0.3302)$ \\
Part orientation $(B)$ & Degree & $0^{\circ}$ & $90^{\circ}$ \\
Raster angle $(C)$ & Degree & $0^{\circ}$ & $45^{\circ}$ \\
Raster width $(D)$ & in./mm & $0.008^{\prime \prime} /(0.2032)$ & $0.022^{\prime \prime} /(0.5588)$ \\
Air gap $(E)$ & in./mm & $-0.0001^{\prime \prime} /(-0.00254)$ & $0.022^{\prime \prime} /(0.5588)$ \\
\hline
\end{tabular}

TABLE 2: Input process parameters that affect output responses.

\begin{tabular}{|c|c|c|c|c|c|c|}
\hline Run & $A$ [layer thickness] & $B$ [part orientation] & $C$ [raster angle] & $D$ [raster width] & $E$ [air gap] & Measured (MPa) \\
\hline 1 & 0.127 & 0 & 0 & 0.2032 & -0.00254 & 32.56 \\
\hline 2 & 0.127 & 0 & 0 & 0.2032 & 0.5588 & 6.52 \\
\hline 3 & 0.127 & 0 & 0 & 0.5588 & -0.00254 & 24.81 \\
\hline 4 & 0.127 & 0 & 0 & 0.5588 & 0.5588 & 10.44 \\
\hline 5 & 0.127 & 0 & 45 & 0.2032 & -0.00254 & 34.61 \\
\hline 6 & 0.127 & 0 & 45 & 0.2032 & 0.5588 & 4.78 \\
\hline 7 & 0.127 & 0 & 45 & 0.5588 & -0.00254 & 30.15 \\
\hline 8 & 0.127 & 0 & 45 & 0.5588 & 0.5588 & 8.08 \\
\hline 9 & 0.127 & 90 & 0 & 0.2032 & -0.00254 & 12.86 \\
\hline 10 & 0.127 & 90 & 0 & 0.2032 & 0.5588 & 4.39 \\
\hline 11 & 0.127 & 90 & 0 & 0.5588 & -0.00254 & 23.55 \\
\hline 12 & 0.127 & 90 & 0 & 0.5588 & 0.5588 & 9.56 \\
\hline 13 & 0.127 & 90 & 45 & 0.2032 & -0.00254 & 15.1 \\
\hline 14 & 0.127 & 90 & 45 & 0.2032 & 0.5588 & 4.01 \\
\hline 15 & 0.127 & 90 & 45 & 0.5588 & -0.00254 & 24.3 \\
\hline 16 & 0.127 & 90 & 45 & 0.5588 & 0.5588 & 8.09 \\
\hline 17 & 0.3302 & 0 & 0 & 0.4572 & -0.00254 & 31.46 \\
\hline 18 & 0.3302 & 0 & 0 & 0.4572 & 0.9652 & 8.43 \\
\hline 19 & 0.3302 & 0 & 0 & 0.9652 & -0.00254 & 24.99 \\
\hline 20 & 0.3302 & 0 & 0 & 0.9652 & 0.9652 & 9.39 \\
\hline 21 & 0.3302 & 0 & 45 & 0.4572 & -0.00254 & 31.42 \\
\hline 22 & 0.3302 & 0 & 45 & 0.4572 & 0.9652 & 7.12 \\
\hline 23 & 0.3302 & 0 & 45 & 0.9652 & -0.00254 & 28.94 \\
\hline 24 & 0.3302 & 0 & 45 & 0.9652 & 0.9652 & 11.07 \\
\hline 25 & 0.3302 & 90 & 0 & 0.4572 & -0.00254 & 20.55 \\
\hline 26 & 0.3302 & 90 & 0 & 0.4572 & 0.9652 & 8.89 \\
\hline 27 & 0.3302 & 90 & 0 & 0.9652 & -0.00254 & 24.72 \\
\hline 28 & 0.3302 & 90 & 0 & 0.9652 & 0.9652 & 11.28 \\
\hline 29 & 0.3302 & 90 & 45 & 0.4572 & -0.00254 & 24.96 \\
\hline 30 & 0.3302 & 90 & 45 & 0.4572 & 0.9652 & 8.82 \\
\hline 31 & 0.3302 & 90 & 45 & 0.9652 & -0.00254 & 27.35 \\
\hline 32 & 0.3302 & 90 & 45 & 0.9652 & 0.9652 & 11.63 \\
\hline
\end{tabular}

using design of experiment (DOE) methodology [19]. The engineering material used for test specimen fabrication is acrylonitrile butadiene styrene (ABS P400). The specimens are fabricated using FDM 400mc machine for respective strength measurement. The main FDM variable parameters are considered in this research in Table 1 to evaluate the correlation between these parameters and the proposed response characteristics. Tests on produced parts are carried out according to the American standard ASTM D638, at an ambient temperature of $23 \pm 2^{\circ} \mathrm{C}$ and a relative humidity $50 \pm 5 \%$.

The rationale for considering the five variable process parameters for experimentation is given here. The layer thickness is known to affect the AM end-product because the smaller the layer thickness the stronger the finished AM part will be when subjected to axial load. Part orientation is important because when the part is built inclined, it will have the tendency to withstand greater loading in 


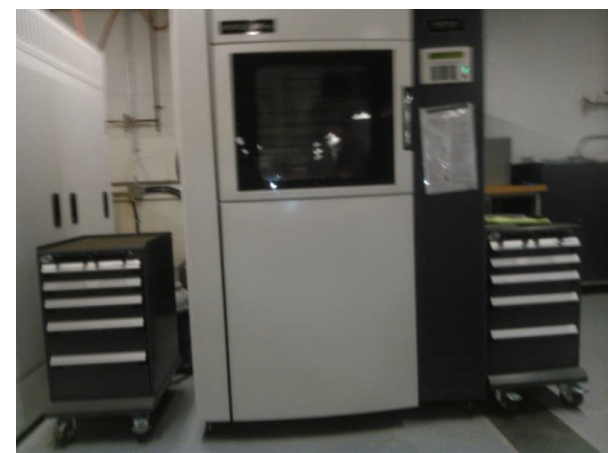

FIGURE 9: FORTUS 400mc System used for the research.

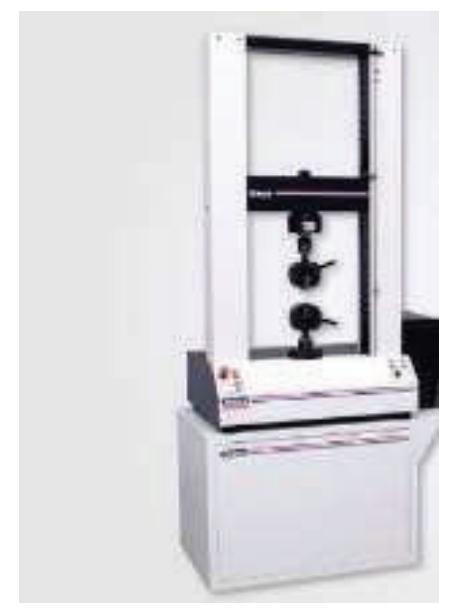

FIGURE 10: UTS System used for the research.

$x$-direction and $y$-direction. Raster angle will have the tendency to affect the internal structure of the finished product. Each neighbouring layer has a raster angle perpendicular to the immediate preceding layer. The raster width is known to affect the finished AM part in such a way that the larger the raster angle the greater the tendency for the finished part to withstand higher tensile stress. Negative air gap is known to produce finished AM part that withstands higher tensile stress. A number of researchers have considered negative air gap and reached these conclusions in their studies similar to our hypothesis. Bagsik and Schöppner [21] investigated the influence of the orientation and the air gap for the manufactured parts based on the mechanical data analysed. The outcome of the investigation was that best results were achieved for all directions by using a negative raster air gap, while with thick filaments better mechanical data was achieved for the $x$ and $z$ build direction, while a thinner filament improves the strength properties for $y$-specimen.

Using DOE approach the 32 full factorial conditions $\left(2^{5}\right)$ were generated as shown in Table 2 for the experimental runs. For each run, three replications were made and averaged. Therefore, the reported measured values are averages of three readings per run. Each run in the design consists of combination of FDM parameters levels and each run result will contain response of tensile strength (UTS). As already

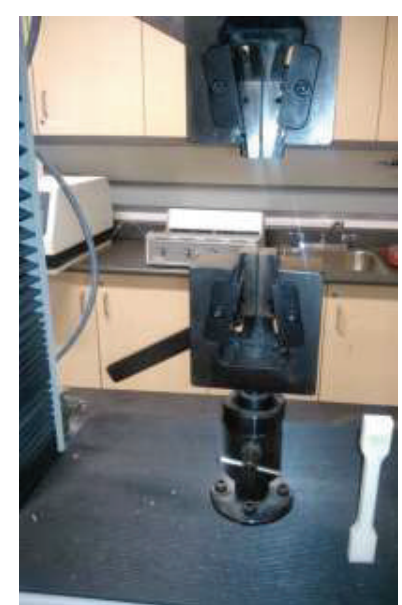

FIGURE 11: Tested tensile specimen (on the right side of UTS machine-bed).

discussed these parameters are clearly described in Figure 9. The parameters are varied to obtain the 32 different AM products. The values for layer thickness, raster width, and air gap given in Table 2 are in millimetre unit since the measured tensile strength (last column) is in $\mathrm{MPa}$.

An image of tested tensile specimen on the table of the UTS machine is shown in Figure 11. The specimen shown is number 14 and this number is marked on the specimen to avoid being mixed up with other specimens. It is important that each specimen is carefully marked after being removed from the FDM machine so that they could be properly identified. Each specimen is $5^{\prime \prime}(127 \mathrm{~mm})$ long, cross-section of $.3750^{\prime \prime} \times .3750^{\prime \prime}(9.53 \mathrm{~mm} \times 9.53 \mathrm{~mm})$, while the middle having a width of $.1875^{\prime \prime}(4.76 \mathrm{~mm})$. Fillet radius is $.0900^{\prime \prime}$ $(2.2 \mathrm{~mm})$.

\section{Group Method for Data Handling (GMDH)}

The framework for modeling chosen for this applied research is based on the Group Method for Data Handling (GMDH) introduced by Ivakhnenko (details are found in [18, 22, 23]) as a means of identifying nonlinear relations between input and output variables. The multilayered iteration (MIA) network is one of the variants of GMDH. The MIA relationship between the inputs and the output of a multiple inputs single output self-organizing network can be represented by an infinite Volterra-Kolmogorov-Gabor (VKG) polynomial of the form $[18,22,23]$

$$
\begin{aligned}
y_{n}= & a_{0}+\sum_{i=1}^{M} a_{i} x_{i}+\sum_{i=1}^{M} \sum_{j=1}^{M} a_{i j} x_{i} x_{j} \\
& +\sum_{i=1}^{M} \sum_{j=1}^{M} \sum_{k=1}^{M} a_{i j k} x_{i} x_{j} x_{k} \cdots,
\end{aligned}
$$


GMDH network

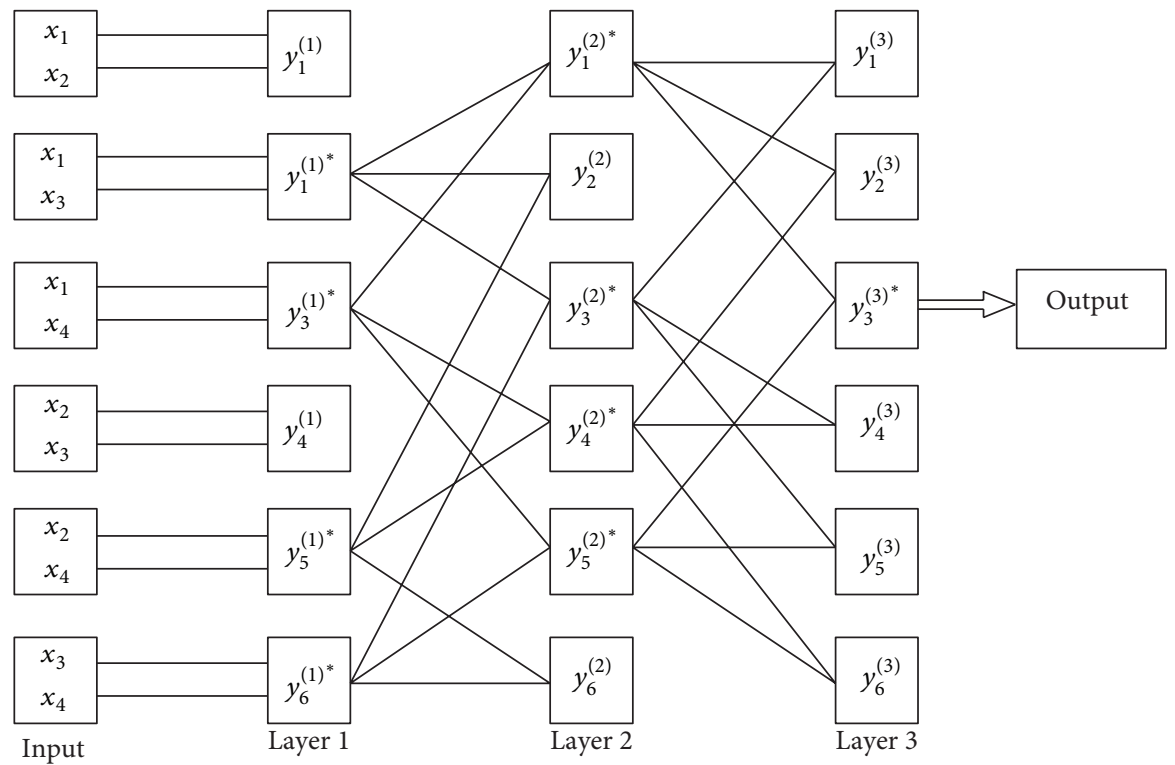

FIGURE 12: GMDH forward feed functional network.

where $X=\left(x_{1}, x_{2}, \ldots, x_{M}\right)$ is the vector of input variables and $A=\left(a_{0}, a_{i}, a_{i j}, a_{i j k}, \ldots\right)$ is the vector of coefficients or weights.

When the GMDH network is completed, there is a set of original inputs that filtered through the layers to the optimal output node. This is the computational network that is to be used in computing predictions (in our application, classifications are implied). The best nodes in the input layer (starred nodes in Figure 12) are retained and form the input to the next layer. The inputs for layer 1 are formed by taking all combinations of the surviving output approximations from the input layer nodes. It is seen that at each layer the order of the polynomial approximation is increased by two. Layer 2's best nodes for approximating the system output are retained and form layer 3 inputs. This process is repeated until the current layer's best approximation is inferior to the previous layer's best approximation.

4.1. Advantages of Basic GMDH Technique. The advantage of using pairs of input is that only six weights (coefficients) have to be computed for each neuron. The number of neurons in each layer increases approximately as the square of the number of inputs. During each training cycle, the synaptic weights of each neuron that minimize the error norm between predicted and measured values are computed and those branches that contribute least to the output of the neuron are discarded, the remaining branches being retained and their synaptic weights kept unchanged thereafter. A new layer is subsequently added and the procedure is repeated until the specified termination conditions are met.

There could be summarized that the GMDH-type polynomial networks influence the contemporary artificial neural network algorithms with several other advantages [23]:
(1) they offer adaptive network representations that can be tailored to the given task;

(2) they learn the weights rapidly in a single step by standard ordinary least square (OLS) fitting which eliminates the need to search for their values and which guarantees finding locally good weights due to the reliability of the fitting technique;

(3) these polynomial networks feature sparse connectivity which means that the best discovered networks can be trained fast.

4.2. Limitations of GMDH Technique. Although standard GMDH provides for a systematic procedure of system modeling and prediction, it has also a number of shortcomings. Anastasakis and Mort [24] have carried out a comprehensive study of the shortcomings of GMDH.

4.2.1. Selection of Input Arguments. One of the main features of GMDH is its ability to objectively select the most appropriate input arguments amongst a set of candidates. However, the identification of these candidate input arguments is not straightforward and may affect its performance.

4.2.2. Inaccuracies in Parameter Estimation. The method of least square estimates is the most popular method to calculate the coefficients of partial descriptions. If the data matrix is well defined its estimates will be accurate; however, in the majority of real world systems the data matrix is ill-defined and the least squares are biased.

4.2.3. Multicollinearity. Another problem found exclusively in multilayer algorithm, which affects the stability of coefficients, is that of multicollinearity. 
4.2.4. Reduction of Complexity. Another shortcoming found GMDH approach is a tendency to generate quite complex polynomial (since the complexity of the network increases with each training and selection cycle through addition of new layers) for relatively simple systems (data input); also, an inclination to producing overly complex network (model) when dealing with highly nonlinear systems owing to its limited generic structure (quadratic two-variable polynomial).

4.2.5. Formulas of Partial Descriptions. Despite the wide range of partial descriptions majority of researchers follow the argument that Volterra series are capable of identifying any nonlinear system and therefore have adopted polynomial partial descriptions similar to Ivakhnenko polynomial. However, due to the complexity of the model and the requirement of including the theory behind the object, many modifications have been designed in order to adapt to system's properties.

4.2.6. Over Fitting. A consequence of complexity is the overfitting problem and poor generalization.

4.2.7. Partition of Data. The objectiveness of GMDH algorithm is based on the utilization of an external criterion to select the optimum model, which requires the partition of the data.

4.2.8. Low Accuracy in GMDH Method. In many cases and particularly in applications of long range prediction the GMDH has been observed to be inaccurate.

4.3. Hybrid-GMDH Network. Based on the shortcomings of the basic GMDH, hybrids of GMDH were proposed to significantly enhance the performance of GMDH [24]. For ease of reference and clarity to readers, the main design steps for the hybrid group method for data handling-genetic algorithm (GMDH-GA) based approach used for the work reported in this paper which applies to similar hybrid-types are summarized here (interested readers may refer to [25] for details on hybrid-GMDH).

Step 1 (configuration of input variable). The system input variables shown in Table 1 are the layer thickness (a), part orientation (b), raster angle (c), raster width (d), and air gap (e).

Step 2 (form training and testing data). Three-quarters of the dataset was used in designing the training data, while the remaining one-quarter was used in designing the testing data.

Step 3 (decision of initial information for constructing the hybrid GMDH-GA structure). The number of generations, population size, crossover rate, and mutation rate were chosen as $25,50,0.9$, and 0.1 , respectively.

Step 4 (determine polynomial neuron (PN) structure). The vector number of input variables of two, the polynomial order of Type 2 (quadratic), and the input variables were assigned to each node of the corresponding layer.

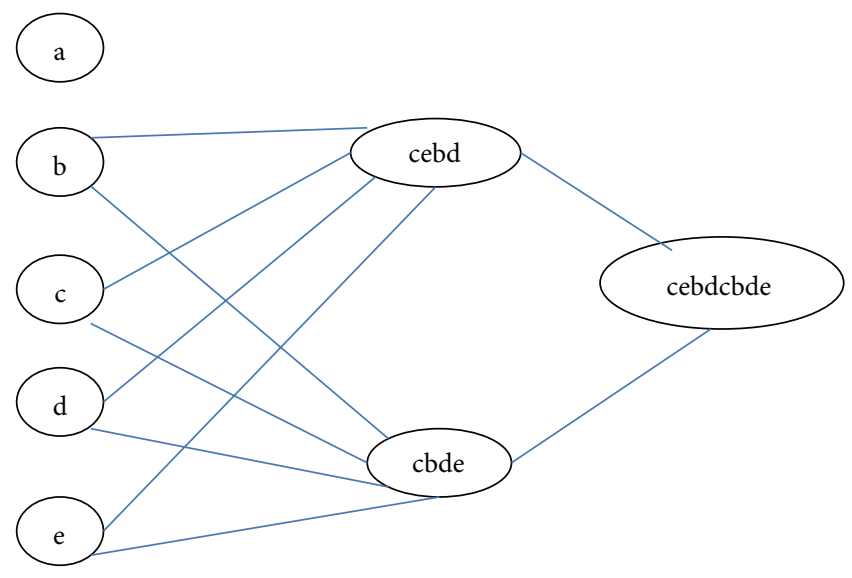

FIGURE 13: Hybrid GMDH-GA structural network for 5-input process parameters.

Step 5 (parametric optimization). Coefficient estimation of the polynomial corresponding to the selected node (PN): the vector of the coefficients of the partial descriptors (PDs) is determined using a standard mean square error for the training dataset subsets.

Step 6 (structural optimization). Select nodes (PNs) with the best predictive capability, and construct their corresponding layer: all nodes of the corresponding layer of the hybrid GMDH-GA architecture are constructed by optimization (see Figure 13).

Step 7 (termination criterion). After the iteration process, the final generation of population consists of highly fit solution population that provides optimum solutions.

\section{GMDH-Based Predictive Model of Tensile Strength}

The process parameters $A, B, C, D$, and $E$ and tensile response of Table 2 were submitted to the MATLAB coded hybrid GMDH-GA software system to develop predictive model that relates the tensile strength to the process parameters. The last column of Table 3 shows the predicted values obtained from the GMDH modelling system, while the second to the last column shows the experimental values. Figure 14 shows the experimental (measured) and predicted values in a graphical form for the thirty two runs. As could be observed our GMDH modelling system predicts very well the behaviour of the tensile strength response with very little deviation.

The GMDH model for the part produced by the Stratasys FDM $400 \mathrm{mc}$ System based on Table 2 is

$$
\begin{aligned}
y_{\mathrm{UTS}}= & 15.3577+54.622 x_{1}+0 x_{2}+0 x_{3}+20.4923 x_{4} \\
& -47.627 x_{5}-0.1122 x_{1} x_{2}+0.0774 x_{1} x_{3} \\
& -37.5299 x_{1} x_{4}+18.6233 x_{1} x_{5}-0.0001 x_{2} x_{3} \\
& +0.0996 x_{2} x_{4}+0.0801 x_{3} x_{5}+0.0066 x_{3} x_{4}
\end{aligned}
$$


TABLE 3: Input process parameters that affect output responses.

\begin{tabular}{|c|c|c|c|c|c|c|c|}
\hline Run & $A$ [layer thickness] & $B$ [part orientation] & $C$ [raster angle] & $D$ [raster width] & $E$ [air gap] & Measured (MPa) & Predicted $(\mathrm{MPa})$ \\
\hline 1 & 0.127 & 0 & 0 & 0.2032 & -0.00254 & 32.56 & 27.76 \\
\hline 2 & 0.127 & 0 & 0 & 0.2032 & 0.5588 & 6.52 & 4.74 \\
\hline 3 & 0.127 & 0 & 0 & 0.5588 & -0.00254 & 24.81 & 28.14 \\
\hline 4 & 0.127 & 0 & 0 & 0.5588 & 0.5588 & 10.44 & 8.58 \\
\hline 5 & 0.127 & 0 & 45 & 0.2032 & -0.00254 & 34.61 & 30.96 \\
\hline 6 & 0.127 & 0 & 45 & 0.2032 & 0.5588 & 4.78 & 4.39 \\
\hline 7 & 0.127 & 0 & 45 & 0.5588 & -0.00254 & 30.15 & 31.96 \\
\hline 8 & 0.127 & 0 & 45 & 0.5588 & 0.5588 & 8.08 & 8.84 \\
\hline 9 & 0.127 & 90 & 0 & 0.2032 & -0.00254 & 12.86 & 12.60 \\
\hline 10 & 0.127 & 90 & 0 & 0.2032 & 0.5588 & 4.39 & 5.10 \\
\hline 11 & 0.127 & 90 & 0 & 0.5588 & -0.00254 & 23.55 & 23.89 \\
\hline 12 & 0.127 & 90 & 0 & 0.5588 & 0.5588 & 9.56 & 8.58 \\
\hline 13 & 0.127 & 90 & 45 & 0.2032 & -0.00254 & 15.1 & 14.33 \\
\hline 14 & 0.127 & 90 & 45 & 0.2032 & 0.5588 & 4.01 & 4.96 \\
\hline 15 & 0.127 & 90 & 45 & 0.5588 & -0.00254 & 24.3 & 26.67 \\
\hline 16 & 0.127 & 90 & 45 & 0.5588 & 0.5588 & 8.09 & 8.78 \\
\hline 17 & 0.3302 & 0 & 0 & 0.4572 & -0.00254 & 31.46 & 28.32 \\
\hline 18 & 0.3302 & 0 & 0 & 0.4572 & 0.9652 & 8.43 & 9.19 \\
\hline 19 & 0.3302 & 0 & 0 & 0.9652 & -0.00254 & 24.99 & 25.85 \\
\hline 20 & 0.3302 & 0 & 0 & 0.9652 & 0.9652 & 9.39 & 11.35 \\
\hline 21 & 0.3302 & 0 & 45 & 0.4572 & -0.00254 & 31.42 & 32.05 \\
\hline 22 & 0.3302 & 0 & 45 & 0.4572 & 0.9652 & 7.12 & 9.06 \\
\hline 23 & 0.3302 & 0 & 45 & 0.9652 & -0.00254 & 28.94 & 29.42 \\
\hline 24 & 0.3302 & 0 & 45 & 0.9652 & 0.9652 & 11.07 & 10.96 \\
\hline 25 & 0.3302 & 90 & 0 & 0.4572 & -0.00254 & 20.55 & 22.30 \\
\hline 26 & 0.3302 & 90 & 0 & 0.4572 & 0.9652 & 8.89 & 8.64 \\
\hline 27 & 0.3302 & 90 & 0 & 0.9652 & -0.00254 & 24.72 & 25.14 \\
\hline 28 & 0.3302 & 90 & 0 & 0.9652 & 0.9652 & 11.28 & 10.53 \\
\hline 29 & 0.3302 & 90 & 45 & 0.4572 & -0.00254 & 24.96 & 24.92 \\
\hline 30 & 0.3302 & 90 & 45 & 0.4572 & 0.9652 & 8.82 & 8.58 \\
\hline 31 & 0.3302 & 90 & 45 & 0.9652 & -0.00254 & 27.35 & 27.85 \\
\hline 32 & 0.3302 & 90 & 45 & 0.9652 & 0.9652 & 11.63 & 10.34 \\
\hline
\end{tabular}

$$
\begin{aligned}
& -0.0899 x_{3} x_{5}-2.7021 x_{4} x_{5}-60.0811 x_{1}^{2} \\
& -0.0011 x_{2}^{2}+0.0012 x_{3}^{2}-6.874 x_{4}^{2}+24.7908 x_{5}^{2},
\end{aligned}
$$

where $x_{1}=A$ (layer thickness); $x_{2}=B$ (part orientation); $x_{3}=C$ (raster angle); $x_{4}=D$ (raster width); $x_{5}=E$ (air gap).

In order to assess the quality of the model realized for the work reported in this paper, some statistical data are given. The sum of square residual, SS: residual: $J=0.4525$; the sum of square deviation, SS: deviation from mean: $S=$ $9.6918 e+03$; hence the coefficient of determination given as $1-J / S$ is given as $r$-squared value: $r^{2}=0.99995$. The regression sum of square, SS_regression $=0.00249$; the error sum of square, SS_error $=6.1089 e-27$; the mean square regression, MSE_regression $=4.9999 e-04$; the mean square error, MSE_error $=2.1817 e-28$; the $F$-value $=2.2917 e+24$.
Figure 15 shows the surface plot of the process parameters for the runs 1-4 while Figure 16 shows the surface plot of the process parameters for the runs 1-32, confirming that the modelling search space is highly complex.

5.1. Examining the Breakage Modes of Specimens. Specimens 13 and 29 (left-most); 3 and 19 (middle); 5 and 21 (right-most) in Figures 15-17 were examined. It is noticed that each pack has three specimens used for experimentation.

The slice height of number 13 is $0.005^{\prime \prime}(0.127 \mathrm{~mm})$ while that of number 29 is $0.139^{\prime \prime}(0.3302 \mathrm{~mm})$, both printed with their longitudinal axis vertical; the raster angle for both is $45^{\circ}$ while the raster widths are $0.008^{\prime \prime}(0.2032 \mathrm{~mm})$ and $0.018^{\prime \prime}(0.4572 \mathrm{~mm})$, respectively. The air gap is the same for both specimens $\left(-0.001^{\prime \prime}\right.$ or $\left.-0.00254 \mathrm{~mm}\right)$. The broken areas confirm the internal structures represented in Table 3. 


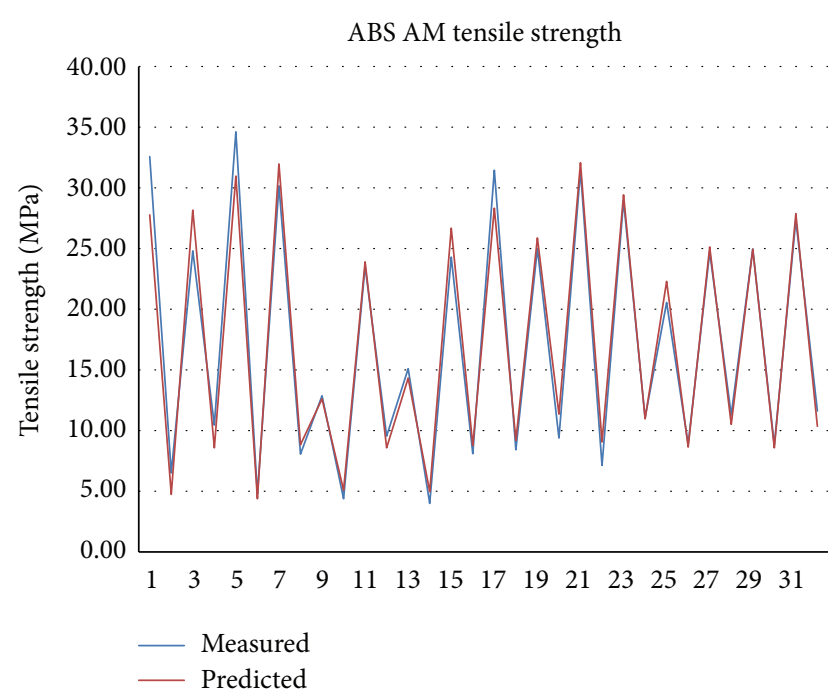

FIGURE 14: Measured and predicted tensile strength response.

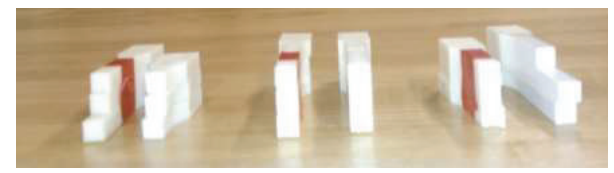

FIGURE 15: Specimens 13 and 29 (left-most); 3 and 19 (middle); 5 and 21 (right-most).

Breakage took place virtually perpendicular to the direction of application of load.

The slice height of number 3 is $0.005^{\prime \prime}(0.127 \mathrm{~mm})$ while that of number 19 is $0.139^{\prime \prime}(0.3302 \mathrm{~mm})$, both printed with their longitudinal axis vertical; the raster angle for both is $0^{\circ}$ while the raster widths are $0.022^{\prime \prime}(0.5588 \mathrm{~mm})$ and $0.038^{\prime \prime}(0.965 \mathrm{~mm})$, respectively. The air gap is the same for both specimens $\left(-0.001^{\prime \prime}\right.$ or $\left.-0.00254 \mathrm{~mm}\right)$. The broken areas confirm the internal structures represented in Table 3. Breakage took place virtually perpendicular to the direction of application of load, in a very neat manner.

The parameters for numbers 5 and 21 are, respectively, the same as those for numbers 13 and 29 , respectively, except that they are printed with the longitudinal axis horizontal. Breakage surface for number 5 is uneven (in a ridge form); this specimen has the largest tensile strength for all the specimens tested. Specimen number 21 shows slight uneven breakage surface.

\section{Optimization of Process Parameters for Tensile Strength Prediction}

6.1. Mathematical Formulation of the Tensile Strength Problem. The hybrid GMDH tensile strength model developed was utilized by the continuous $\mathrm{DE}$ for optimization in order to determine the optimal combinations of layer thickness $(A)$, part orientation $(B)$, raster angle $(C)$, raster width $(D)$, and air gap $(E)$ that result in maximizing tensile strength for the FDM

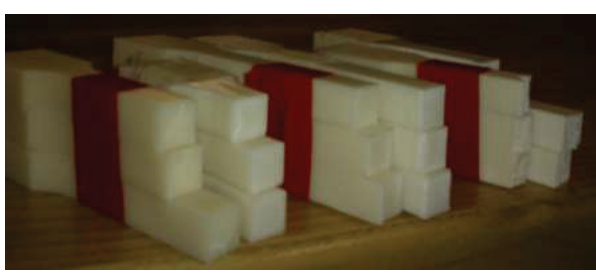

FIGURE 16: Specimens as in Figure 15 but with more exposure of broken surfaces.

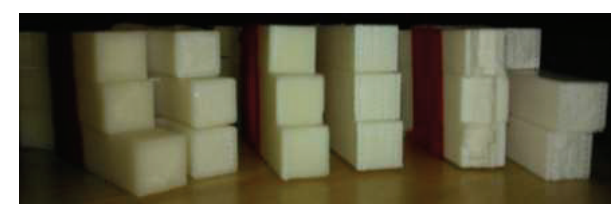

FIgURE 17: Specimens as in Figure 15 but with most exposure of broken surfaces.

part. The optimization problem is essentially that of using (2), as objective function with constraints taken from Table 2 .

The tensile strength optimization problem can now be fully mathematically stated as follows:

$$
\begin{aligned}
y_{\mathrm{UTS}}= & 15.3577+54.622 x_{1}+0 x_{2}+0 x_{3} \\
& +20.4923 x_{4}-47.627 x_{5}-0.1122 x_{1} x_{2} \\
& +0.0774 x_{1} x_{3}-37.5299 x_{1} x_{4}+18.6233 x_{1} x_{5} \\
& -0.0001 x_{2} x_{3}+0.0996 x_{2} x_{4}+0.0801 x_{3} x_{5} \\
& +0.0066 x_{3} x_{4}-0.0899 x_{3} x_{5}-2.7021 x_{4} x_{5} \\
& -60.0811 x_{1}^{2}-0.0011 x_{2}^{2}+0.0012 x_{3}^{2} \\
& -6.874 x_{4}^{2}+24.7908 x_{5}^{2} \\
\text { s.t. } & 0.127 \leq x_{1} \leq 0.3302 \\
& 0 \leq x_{2} \leq 90 \\
& 0 \leq x_{3} \leq 45 \\
& 02034 \leq x_{4} \leq 0.9652 \\
& -0.00254 \leq x_{5} \leq 0.9652 .
\end{aligned}
$$

6.2. Differential Evolution Scheme. The differential evolution (DE) algorithm introduced by Storn and Price [20] is a novel parallel direct search method, which utilizes Np parameter vectors as a population for each generation G. DE is one of the extant evolutionary approaches used to solve complex real-life problems. It was primarily designed for continuous domain space formulation but was reformulated to solve permutative problems by Donald and Onwubolu [27]. The steps involved in the classical DE are summarized as follows:

Step 1: initialization,

Step 2: mutation,

Step 3: crossover, 
TABLE 4: DE control parameters used for experimentation.

\begin{tabular}{lc}
\hline Population size, NP & 50 \\
Number of parameter & 5 \\
Mutation probability, $F$ & 0.20 \\
Crossover probability, CR & 0.60 \\
Number of generations & 500 \\
\hline
\end{tabular}

TABLE 5: Optimal cutting parameters from DE.

\begin{tabular}{lcc}
\hline Tensile strength & $\mathrm{MPa}$ & 32.76 \\
Layer thickness $(A)$ & in./mm & $0.01^{\prime \prime} / 0.2778$ \\
Part orientation $(B)$ & Degree & $9.05^{\circ}$ \\
Raster angle $(C)$ & Degree & $45^{\circ}$ \\
Raster width $(D)$ & in. $/ \mathrm{mm}$ & $0.022^{\prime \prime} /(0.5588)$ \\
Air gap $(E)$ & in. $/ \mathrm{mm}$ & $0.0001^{\prime \prime} /(0.0025)$ \\
\hline
\end{tabular}

Step 4: selection,

Step 5: stopping criteria.

Using DE, which is one of the extant evolutionary approaches, the optimal parameter settings for the FDM tensile test were found. DE was used for optimizing the tensile strength optimization problem expressed in (3) and (4). The control conditions used for optimization are shown in Table 4. The approach of using the hybrid GMDH model (3), as objective functions with constraints given in (4) for optimizing the tensile strength problem, is more straightforward than when ANN is employed in modeling. This type of mathematical formulation makes the $\mathrm{GMDH}$ response models to be more useful to the end-user since the models for the problem being solved are transparent and could be used for future applications. Moreover, the mathematical models are easy to be used as the objective functions by most standard optimization techniques for determining optimal cutting and response conditions.

For the experimentation, the optimal process parameters and tensile strength optimization problem that the DE found are given in Table 5.

\section{Results and Discussions}

The results are discussed under specific and general cases. The specific discussion of the results is based on how tensile strength relates with the process parameters of layer thickness, part orientation, raster angle, raster width, and air gap. The general discussions relate to the conditions under which the weakest and strongest AM part is produced.

Effect of Layer Thickness on Tensile Strength. The highest tensile strength occurs for the minimum layer thickness of $0.005^{\prime \prime} /(0.127 \mathrm{~mm})$ on run $5(34.61 \mathrm{MPa})$. The next highest value also occurs for the minimum layer thickness of $0.005^{\prime \prime} /(0.127 \mathrm{~mm})$ in run $1(32.56 \mathrm{MPa})$. These values of tensile strength are greater than the highest tensile strength obtained for the maximum layer thickness of $0.013^{\prime \prime} /(0.3302)$. Consequently, we conclude that the maximum tensile strength occurs when the layer thickness is a minimum.

Effect of Part Orientation on Tensile Strength. Comparing the tensile strength values for run $1(32.56 \mathrm{MPa})$ and run $9(12.86 \mathrm{MPa})$, respectively, run $2(6.52 \mathrm{MPa})$ and run 10 (4.39 $\mathrm{MPa})$, respectively, and so forth, it is observed that part orientation significantly affects the tensile strength of AM parts. The zero angle part orientation is the strongest because the layers are parallel to the direction in which load is applied. Parts that have $90^{\circ}$ orientation during production are the weakest.

Effect of Raster Angle on Tensile Strength. In this case, we compare the strength values for run $1(32.56 \mathrm{MPa})$ and run $5(34.61 \mathrm{MPa})$, respectively, run $2(6.52 \mathrm{MPa})$ and run 6 $(4.78 \mathrm{MPa})$, and so forth. This trend is repeated throughout the entire measured results. Therefore, it is concluded that tensile strength increases with increase in raster angle; howbeit, the increase is not of much magnitude.

Effect of Raster Width on Tensile Strength. The effect of raster width on tensile strength is studied by comparing cases in which only raster width changes. In this case we compare run $1(32.56 \mathrm{MPa})$ and run $3(24.81 \mathrm{MPa})$, respectively, run $5(34.61 \mathrm{MPa})$ and run $5(34.61 \mathrm{MPa})$, respectively,..., run 29 $(24.96 \mathrm{MPa})$ and run $31(27.35 \mathrm{MPa})$, respectively, and run 30 $(8.82 \mathrm{MPa})$ and run $32(11.63 \mathrm{MPa})$, respectively, and so forth. From the observations, we conclude that the tensile strength increases with minimum raster width.

Effect of Air Gap on Tensile Strength. To study the effect of air gap on tensile strength, two consecutive runs are compared: run $1(32.56 \mathrm{MPa})$ and run $2(6.52 \mathrm{MPa})$, respectively, run $3(24.81 \mathrm{MPa})$ and run $4(10.44 \mathrm{MPa})$, and so forth. It is observed that negative air gap results in the strongest AM part.

In general the following observations are made.

(1) The weakest AM parts are produced when air gap is maximum (runs 2, 6, 10, 14, and 16 for layer thickness of $0.005^{\prime \prime} /(0.127)$ and runs $18,20,22,26$, and 30 for layer thickness of $\left.0.013^{\prime \prime} /(0.3302)\right)$

(2) The strongest AM parts are produced with negative air gap (NAG) (runs 1, 3, 5, and 7 for layer thickness of $0.005^{\prime \prime} /(0.127)$ and runs $17,19,21,23,27,29$, and 31 for layer thickness of $\left.0.013^{\prime \prime} /(0.3302)\right)$

However, from our experimentation, for the maximum raster width of 0.022 , an air gap of -0.001 gave very poor quality product, with "over-fill" in which the printed material spilled beyond the expected positions. The air gap was reduced to -0.0001 to give good quality results. To get good results, the percentage of the air gap to the maximum raster width should be $0.45 \%((0.0001 / 0.022) * 100)$. 


\section{Conclusions}

In this work, functional relationship between process parameters and tensile strength for FDM process has been developed using group method for data modelling for prediction purpose. An initial test was carried out to determine whether part orientation and raster angle variations affect the tensile strength. It was found that both process parameters affect tensile strength response. For the more elaborate experimentation, the process parameters considered are layer thickness, orientation, raster angle, raster width, and air gap. The process parameters and the experimental results were submitted to a hybrid GMDH-GA system, resulting in predicted output, in which the predicted output values were found to correlate very closely with the measured values.

Since FDM process is a complex one, it is really challenging to determine good functional relationship between responses and process parameters. Using differential evolution (DE), which is one of the extant evolutionary approaches, optimal parameter settings were found. Our investigations have shown the following.

(1) Minimum layer thickness improves tensile strength, although is more costly due to more material usage for manufacturing parts.

(2) Negative air gap significantly improves the tensile strength.

(3) Minimum raster widths also improve tensile strength.

(4) Part orientation plays a major role as could be observed from the results. For zero part orientation (with the part orientation coinciding with the direction of tensile loading), maximum tensile strength is obtained.

(5) Increased raster angle also improves tensile strength, although not very significantly. The optimized solutions that DE found agree very reasonably with our observations from Table 2.

(6) Our experimental results in Table 2 show that maximum tensile strength is obtained in run 5 for which the part orientation is zero and raster angle and raster width are maximum, with negative air gap. DE optimal solutions match these observations, with part orientation being $9.05^{\circ}$.

Consequently, the conclusions reached in this research are reliable and can be applied to real life applications. Future work will include the investigation of other mechanical properties such as compressive strength and torsional strength. Microstructure investigation of broken parts during testing will be done using high powered microscope.

\section{Nomenclature}

$x_{1}: A$ (layer thickness)

$x_{2}: B$ (part orientation)

$x_{3}$ : $C$ (raster angle)

$x_{4}: D$ (raster width)

$x_{5}: E$ (air gap).

\section{Conflict of Interests}

The authors declare that there is no conflict of interests regarding the publication of this paper.

\section{Acknowledgments}

The two technologists Attila Nagy and Andrew Orton in the Sheridan Centre for Advanced Manufacturing \& Design Technology (CAMDT) assisted the authors with the AM lab procedures, and they are gratefully appreciated.

\section{References}

[1] I. Gibson, D. W. Rosen, and B. Stucker, Additive Manufacturing Technologies: Rapid Prototyping to Direct Digital Manufacturing, Springer, Heidelberg, Germany, 2010.

[2] N. Hopkinson, R. J. M. Hagur, and P. H. Dickens, Rapid Manufacturing: An Industrial Revolution for the Digital Age, John Wiley \& Sons, London, UK, 2006.

[3] B. Wiedemann and H.-A. Jantzen, "Strategies and applications for rapid product and process development in Daimler-Benz AG," Computers in Industry, vol. 39, no. 1, pp. 11-25, 1999.

[4] S. Upcraft and R. Fletcher, "The rapid prototyping technologies," Assembly Automation, vol. 23, no. 4, pp. 318-330, 2003.

[5] S. Mansour and R. Hague, "Impact of rapid manufacturing on design for manufacture for injection moulding," Proceedings of the Institution of Mechanical Engineers, Part B: Journal of Engineering Manufacture, vol. 217, no. 4, pp. 453-461, 2003.

[6] G. N. Levy, R. Schindel, and J. P. Kruth, "Rapid manufacturing and rapid tooling with layer manufacturing (LM) technologies, state of the art and future perspectives," CIRP Annals: Manufacturing Technology, vol. 52, no. 2, pp. 589-609, 2003.

[7] O. Ivanova, C. Williams, and T. Campbell, "Additive manufacturing (AM) and nanotechnology: promises and challenges," Rapid Prototyping Journal, vol. 19, no. 5, pp. 353-364, 2013.

[8] P. K. Jain, P. M. Pandey, and P. V. M. Rao, "Effect of delay time on part strength in selective laser sintering," International Journal of Advanced Manufacturing Technology, vol. 43, no. 1-2, pp. 117126, 2009.

[9] K. Chockalingam, N. Jawahar, and U. Chandrasekhar, "Influence of layer thickness on mechanical properties in stereolithography," Rapid Prototyping Journal, vol. 12, no. 2, pp. 106113, 2006.

[10] J. F. Rodríguez, J. P. Thomas, and J. E. Renaud, "Mechanical behavior of acrylonitrile butadiene styrene (ABS) fused deposition materials. Experimental investigation," Rapid Prototyping Journal, vol. 7, no. 3, pp. 148-158, 2001.

[11] S.-H. Ahn, M. Montero, D. Odell, S. Roundy, and P. K. Wright, "Anisotropic material properties of fused deposition modeling ABS," Rapid Prototyping Journal, vol. 8, no. 4, pp. 248-257, 2002.

[12] A. K. Sood, R. K. Ohdar, and S. S. Mahapatra, "Parametric appraisal of mechanical property of fused deposition modelling processed parts," Materials and Design, vol. 31, no. 1, pp. 287$295,2010$.

[13] A. K. Sood, R. K. Ohdar, and S. S. Mahapatra, "Experimental investigation and empirical modelling of FDM process for compressive strength improvement," Journal of Advanced Research, vol. 3, no. 1, pp. 81-90, 2012.

[14] B. H. Lee, J. Abdullah, and Z. A. Khan, "Optimization of rapid prototyping parameters for production of flexible ABS object," 
Journal of Materials Processing Technology, vol. 169, no. 1, pp. 5461, 2005.

[15] C. S. Lee, S. G. Kim, H. J. Kim, and S. H. Ahn, "Measurement of anisotropic compressive strength of rapid prototyping parts," Journal of Materials Processing Technology, vol. 187-188, pp. 627630, 2007.

[16] S. K. Panda, S. Padheee, A. K. Sood, and S. S. Mahapatra, "Optimization of fused deposition modelling (FDM) process parameters using bacterial foraging technique," Intelligent Information Management, vol. 1, pp. 89-97, 2009.

[17] K. Thrimurthulu, P. M. Pandey, and N. V. Reddy, "Optimum part deposition orientation in fused deposition modeling," International Journal of Machine Tools and Manufacture, vol. 44, no. 6, pp. 585-594, 2004.

[18] H. R. Madala and A. G. Ivakhnenko, Inductive Learning Algorithms for Complex Systems Modelling, CRC Press, Boca Raton, Fla, USA, 1994.

[19] D. C. Montgomery, Design and Analysis of Experiments, John Wiley \& Sons, Singapore, 5th edition, 2003.

[20] R. Storn and K. Price, "Differential evolution-a simple and efficient heuristic for global optimization over continuous spaces," Journal of Global Optimization, vol. 11, no. 4, pp. 341359, 1997.

[21] A. Bagsik and V. Schöppner, "Mechanical properties of fused deposition modeling parts manufactured with Ultem $* 9085$," in Proceedings of the 69th Annual Technical Conference of the Society of Plastics Engineers (ANTEC '11), pp. 1294-1298, Boston, Mass, USA, May 2011.

[22] A. G. Ivakhnenko, "Polynomial theory of complex systems," IEEE Transactions on Systems, Man and Cybernetics, vol. SMC-1, no. 4, pp. 364-378, 1971.

[23] N. Y. Nikolaev and H. Iba, "Polynomial harmonic GMDH learning networks for time series modeling," Neural Networks, vol. 16, no. 10, pp. 1527-1540, 2003.

[24] L. Anastasakis and N. Mort, “The development of self-organization techniques in modelling: a review of the group method of data handling (GMDH)," Research Report 813, Department of Automatic Control \& Systems Engineering, The University of Sheffield, Sheffield, UK, 2001.

[25] G. C. Onwubolu, Ed., Onwubolu Hybrid Self-Organizing Modeling Systems, Springer, 2009.

[26] G. C. Onwubolu, "Selection of drilling operations parameters for optimal tool loading using integrated response surface methodology: a Tribes approach," International Journal of Production Research, vol. 44, no. 5, pp. 959-980, 2006.

[27] G. C. Onwubolu and D. Davendra, Eds., Differential Evolution: A Handbook for Global Permutation-Based Combinatorial Optimization, Studies in Computational Intelligence, Springer, New York, NY, USA, 2009. 

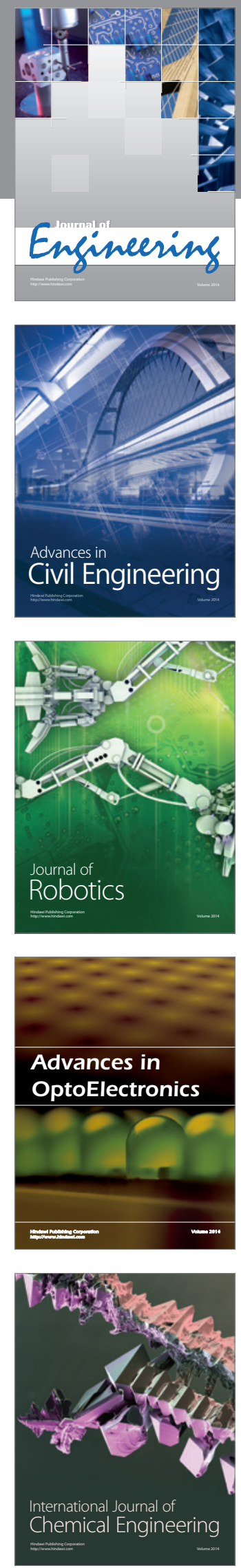

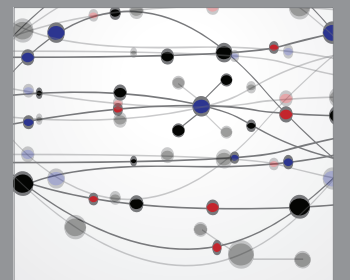

The Scientific World Journal
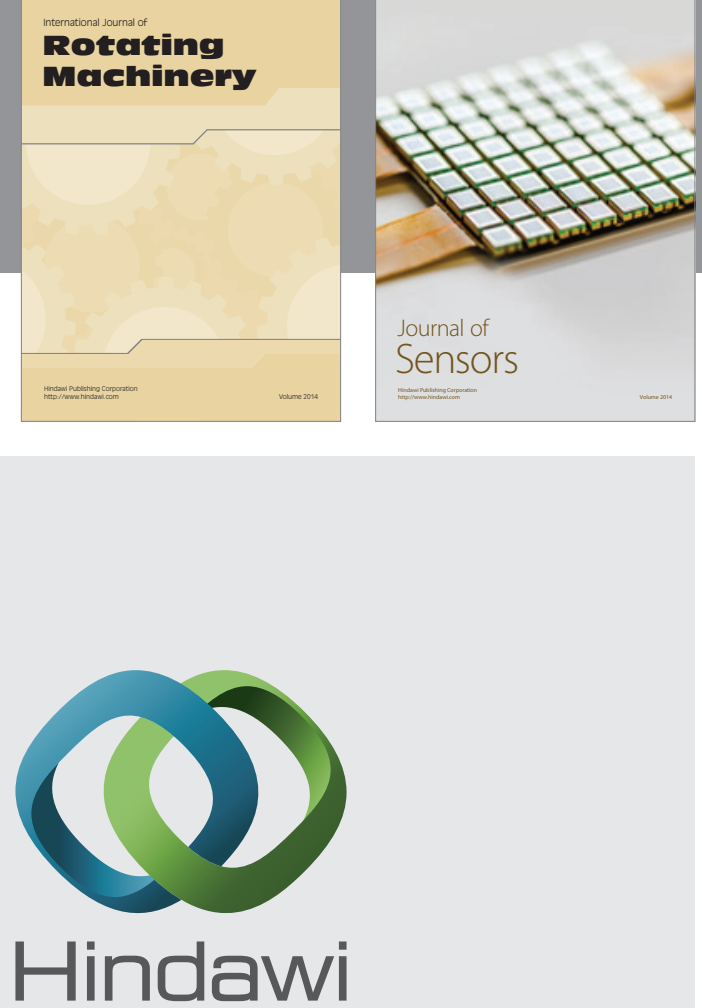

Submit your manuscripts at http://www.hindawi.com
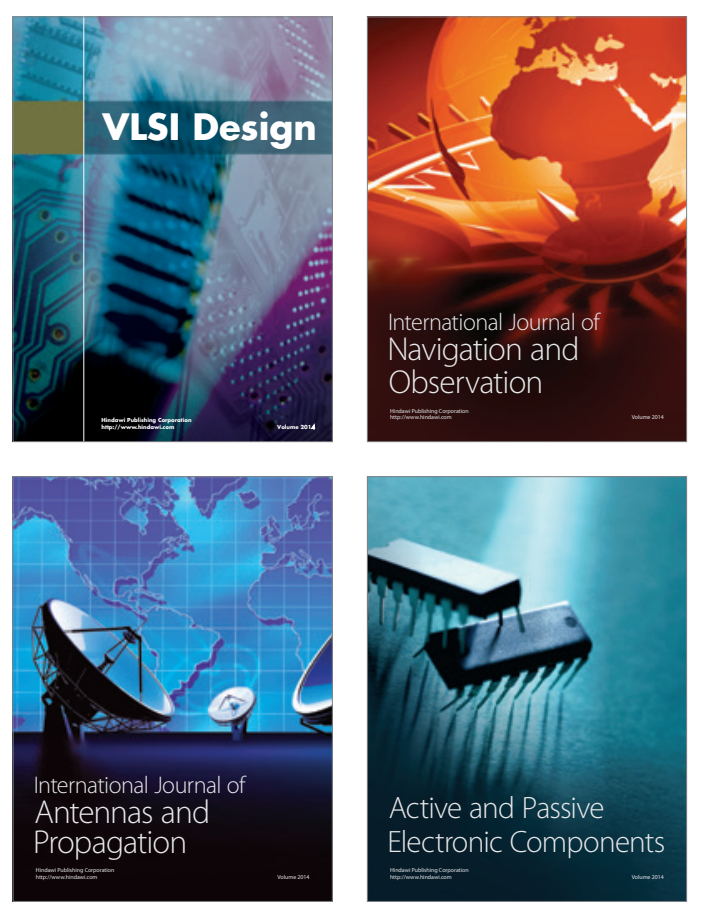
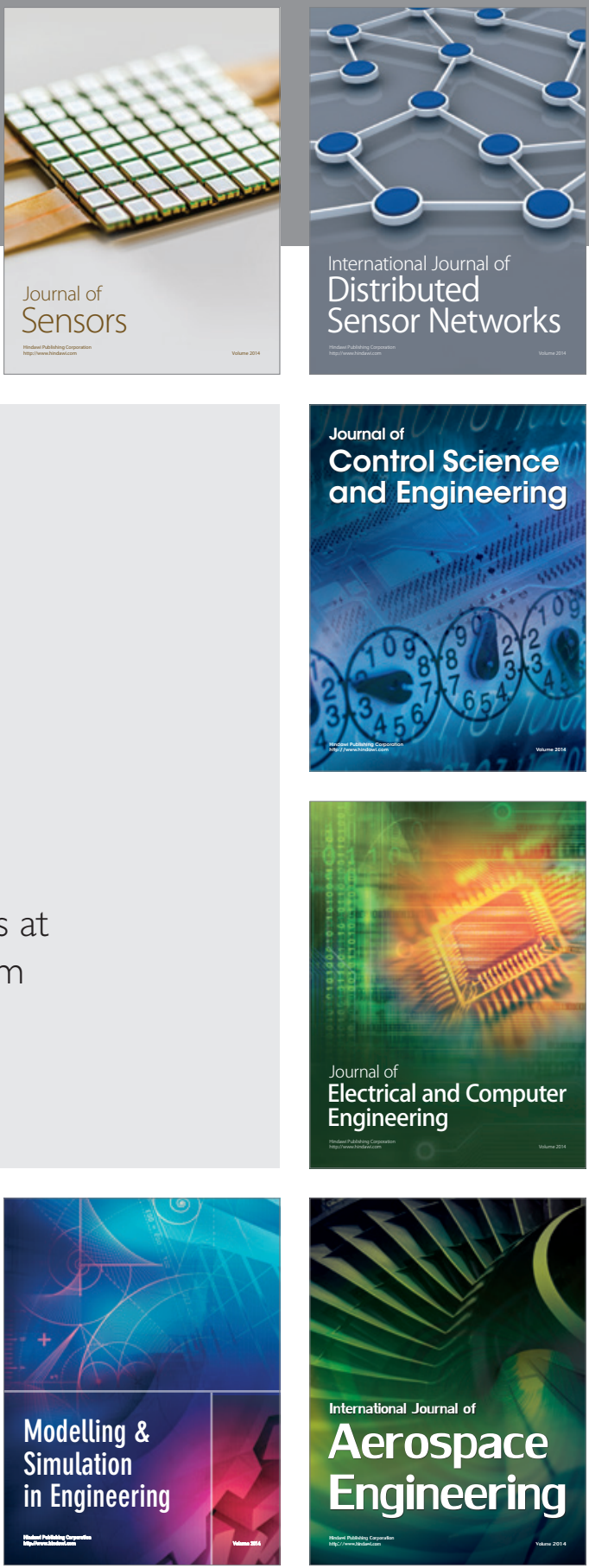

Journal of

Control Science

and Engineering
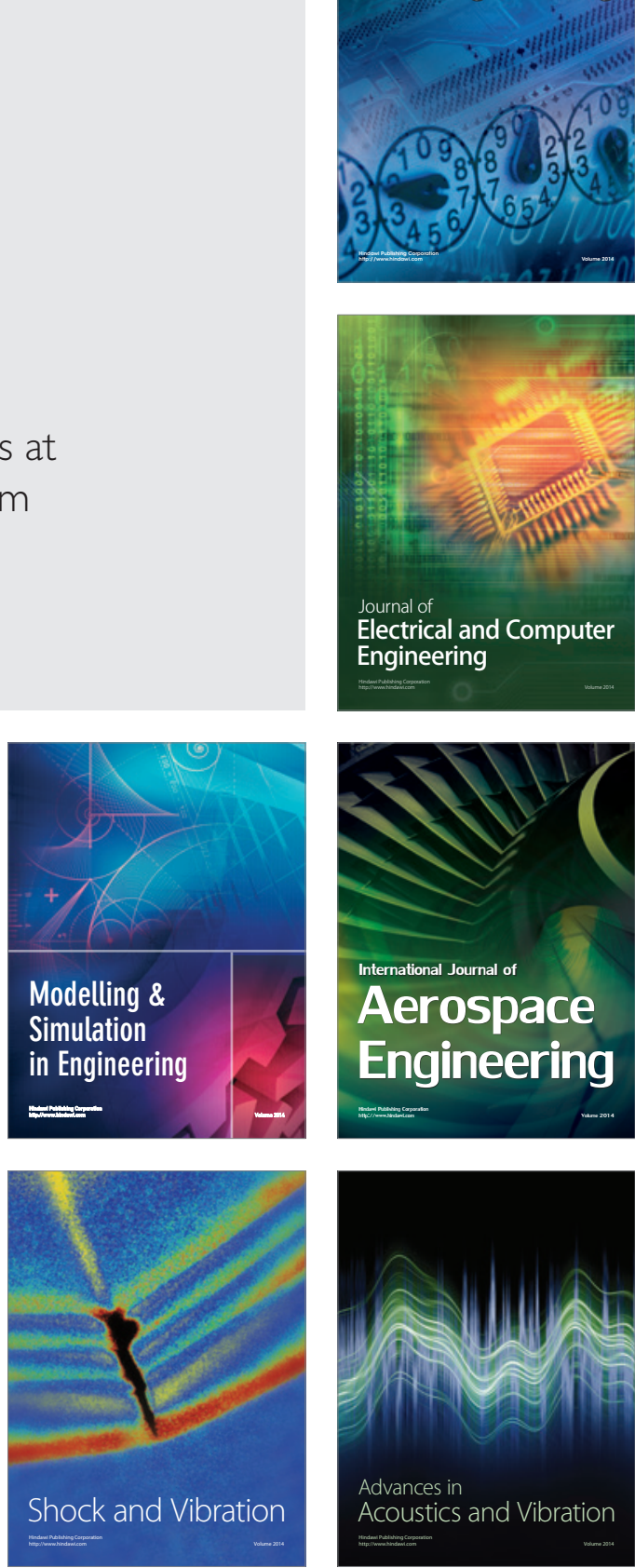